\title{
ВMJ Global Health Indirect effects of COVID-19 on maternal, neonatal, child, sexual and reproductive health services in Kampala, Uganda
}

Jessica Florence Burt, ${ }^{1,2}$ Joseph Ouma, ${ }^{2}$ Lawrence Lubyayi, ${ }^{3}$ Alexander Amone, ${ }^{2}$ Lorna Aol, ${ }^{2}$ Musa Sekikubo, ${ }^{4}$ Annettee Nakimuli, ${ }^{4}$ Eve Nakabembe, ${ }^{4}$ Robert Mboizi, ${ }^{2}$ Philippa Musoke, ${ }^{2}$ Mary Kyohere, ${ }^{2}$ Emily Namara Lugolobi, ${ }^{2}$ Asma Khalil, ${ }^{5}$ Kirsty Le Doare ${ }^{3,5}$

To cite: Burt JF, Ouma J, Lubyayi L, et al. Indirect effects of COVID-19 on maternal, neonatal, child, sexual and reproductive health services in Kampala, Uganda. BMJ Global Health 2021;6:e006102. doi:10.1136/ bmjgh-2021-006102

Handling editor Sanni Yaya

- Additional supplemental material is published online only. To view, please visit the journal online (http://dx.doi.org/10. 1136/bmjgh-2021-006102)

Received 23 April 2021 Accepted 8 August 2021
Check for updates

(c) Author(s) (or their employer(s)) 2021. Re-use permitted under CC BY-NC. No commercial re-use. See rights and permissions. Published by BMJ.

For numbered affiliations see end of article.

Correspondence to Professor Kirsty Le Doare; kiledoar@sgul.ac.uk

\section{ABSTRACT}

Background COVID-19 impacted global maternal, neonatal and child health outcomes. We hypothesised that the early, strict lockdown that restricted individuals' movements in Uganda limited access to services. Methods An observational study, using routinely collected data from Electronic Medical Records, was carried out, in Kawempe district, Kampala. An interrupted time series analysis assessed the impact on maternal, neonatal, child, sexual and reproductive health services from July 2019 to December 2020. Descriptive statistics summarised the main outcomes before (July 2019-March 2020), during (April 2020-June 2020) and after the national lockdown (July 2020-December 2020).

Results Between 1 July 2019 and 31 December 2020, there were 14401 antenatal clinic, 33499 deliveries, 111658 childhood service and 57174 sexual health attendances. All antenatal and vaccination services ceased in lockdown for 4 weeks.

During the 3-month lockdown, the number of antenatal attendances significantly decreased and remain below pre-COVID levels (370 fewer/month). Attendances for prevention of mother-to-child transmission of HIV dropped then stabilised. Increases during lockdown and immediately postlockdown included the number of women treated for high blood pressure, eclampsia and pre-eclampsia (218 more/month), adverse pregnancy outcomes (stillbirths, low-birth-weight and premature infant births), the rate of neonatal unit admissions, neonatal deaths and abortions. Maternal mortality remained stable. Immunisation clinic attendance declined while neonatal death rate rose (from 39 to 49/1000 livebirths). The number of children treated for pneumonia, diarrhoea and malaria decreased during lockdown. Conclusion The Ugandan response to COVID-19 negatively impacted maternal, child and neonatal health, with an increase seen in pregnancy complications and fetal and infant outcomes, likely due to delayed care-seeking behaviour. Decreased vaccination clinic attendance leaves a cohort of infants unprotected, affecting all vaccinepreventable diseases. Future pandemic responses must consider impacts of movement restrictions and access to preventative services to protect maternal and child health.

\section{Key questions}

What is already known?

- The response to COVID-19 has been shown to have indirectly impacted on maternal, child, neonatal, sexual and reproductive health.

- This is largely related to access to services and fear of contracting COVID-19 in outpatient departments.

- There has been very little data published on the health impacts of the COVID-19 response in Uganda.

What are the new findings?

- Antenatal attendances decreased dramatically in April, followed by increased numbers of low-birthweight infants and neonatal deaths.

- Newborn immunisations against polio, tetanus, diphtheria, hepatitis B, Haemophilus influenzae, rotavirus and pneumococcus decreased significantly.

- Sexual and reproductive health services were reduced in number.

What do the new findings imply?

- Although Uganda has been less affected directly by COVID-19 infections in the first wave, the indirect impacts are far-reaching and will have future influences on population health.

- There is a degree of resilience within the healthcare service, but many services were adversely affected by the lockdown leading to poorer pregnancy and neonatal outcomes.

- Antenatal and vaccination services are of particular importance in ensuring the safety of mother and child and must be prioritised in the responses to future pandemics.

\section{INTRODUCTION}

Uganda, as with many nations in the WHO Africa region, has largely avoided the considerable infection rate and death toll from COVID-19 that other nations saw during the first wave, ${ }^{12}$ with 84116 confirmed cases and 1966 deaths reported as of 6 July $2021 .^{3}$ While 
this is likely under-representative of the true morbidity and mortality, ${ }^{4}$ Uganda has successfully minimised the spread and direct impact of COVID-19 within its borders through its early, rapid and severe response. However, maternal and child health services were severely impacted by these measures during the height of the lockdown, which may have indirectly affected morbidity and mortality in this group.

\section{Effects of COVID-19}

Symptomatic COVID-19 infection in pregnancy is linked to worse maternal and neonatal outcomes than for pregnancies without COVID-19. ${ }^{5-7}$ Studies across the UK and USA have also shown increased preterm birth, stillbirth, small for gestational age babies and neonatal mortality over the COVID-19 period and in relation to infection in pregnancy. ${ }^{8}$

However, global estimates of the indirect impacts of COVID-19 could amount to up to a $38.6 \%$ increase in maternal mortality, and $44.7 \%$ increase in child mortality per month across 118 low and middle-income countries. ${ }^{9}$ The main factors proposed are disruptions to childbirth services and antenatal care (ANC) such as the management of pre-eclampsia and supplementation advice, wasting and curative child services, ${ }^{9}$ which the WHO has documented as being affected in many locations. ${ }^{10}$ Additionally, disruptions to family planning services including access to contraception and safe abortions will result in an additional rise in maternal deaths, abortion-related complications and a large unmet need for contraceptives. ${ }^{11}$ Further impacts on maternal and child outcomes may be seen through issues surrounding the provision of prevention and management of HIV, ${ }^{9} 12$ reduced lactation support ${ }^{14}$ and conflicting guidance on whether to avoid breast feeding if infected. ${ }^{15}$ These impacts have been reported in some low-resource settings globally, ${ }^{101617}$ particularly with reduced antenatal attendances, linked to transportation restrictions, fear of transmission and lack of antenatal education. ${ }^{17}$

\section{COVID-19 in Uganda}

Preparation and readiness measures against COVID-19 in Uganda began between January and March 2020, focusing on health systems strengthening and capacity building, aided by early allocation of WHO funding. ${ }^{18} 19$ From 2 March, the public were informed of the threat of COVID-19, with education and training subsequently disseminated. ${ }^{18}$ Testing focused on contacts of identified cases and those returning from travel, with populationwide lockdown measures imposed quickly after the first case in Uganda was reported on 21 March 2020. ${ }^{20} 21$ This included border closures, port-of-entry screenings and quarantines for travellers. ${ }^{18}$ By 25 March, this escalated to a ban on group gatherings and non-essential internal travel, recommendation to work from home and close schools. ${ }^{1822}$ The travel restrictions included the cessation of all public transport and a ban on the use of private vehicles without explicit permission to travel. ${ }^{23}$ At a local level, non-essential visits to Kawempe National Referral Hospital (KNRH) were prohibited for a short time (from 23 March 2020 to 21 April 2020), which included the closure of ANC and childhood immunisation clinics. The Ugandan Ministry of Health $(\mathrm{MoH})$ implemented PCRbased screening for symptomatic patients and any patient who was PCR positive was admitted to a dedicated ward.

This paper aims to quantify the indirect impact of COVID-19 on maternal, neonatal and childhood outcomes at KNRH in Kampala.

\section{METHODS}

This was a single-site observational study, which utilised retrospectively collected data, based on KNRH. This is a large, urban hospital with over 21000 deliveries per annum, 200 antenatal clinic visits and 100 child admissions to hospital per day. ${ }^{24}$ The hospital provides preventative and curative care during pregnancy and intrapartum, newborn and postnatal care, a paediatric ward and vaccination services at a standard indicative of care in urban Uganda. After the initial lockdown period ( 4 weeks without outpatient services), measures to reduce the number of women attending ANC included reducing the number of appointments per day from 150 to 90 for $\mathrm{ANC}$ and all women $<26$ weeks gestation being sent away to return after 30 weeks. For infants, the vaccination clinic remained operating routinely. During the initial phases of lockdown (April and May 2020), 35/60 doctors were reassigned to acute care at COVID-19 centres in anticipation of a large number of COVID-19 cases, but 53 nurses were recruited at the same time with result-based financing support raising the number of nurse/midwifes on site from 184 to 237 after April 2020.

\section{Patient and public involvement}

Patients were not involved directly in the formation of this study. We have involved women in a separate, dedicated qualitative study about their experiences of ANC during the pandemic. ${ }^{25}$

\section{Data collection}

Data were retrospectively collected in January 2021, by hospital staff with access to the Electronic Medical Records (EMR) system. This system is part of the Uganda Ministry of Health $(\mathrm{MoH})$ eHealth Policy, Strategy and Implementation Plan and utilises the District Health Information Software 2 (DHIS2).$^{26}$ The DHIS2 indicators for which data were collected are detailed in the available data set $^{27}$ and were taken from health management information system data, which is reported to the $\mathrm{MoH}$, covering pregnancy preventative services, pregnancy curative services, childbirth, care of the newborn, postnatal care, preventative childcare, curative childcare, preventative services for women of reproductive age, curative services for women of reproductive age and unavailability of medicines and commodities. Monthly totals were gathered for the period from July 2019 to December 2020. In accordance with the Sex and Gender 
Equity in Research guidelines, pregnancy, childbirth and sexual health-related indicators are reported for those of the female sex, and no segregation is made between male and female sex or gender for childcare indicators as this was not part of the reporting data. ${ }^{28}$

Neonatal mortality was calculated as the sum of immediate neonatal deaths and deaths from neonatal sepsis 0-7 days, neonatal sepsis 8-28 days, neonatal pneumonia, neonatal meningitis, neonatal jaundice, premature baby (as condition that requires management) and other neonatal conditions.

\section{Statistical analysis}

Data were input into Microsoft Excel and exported to $\mathrm{R}$ V.4.0.4 (R Foundation for Statistical Computing, Vienna, Austria) for further analysis purposes. We calculated the number of attendances per month for each indicator and then analysed the aggregated data at the month level as a proportion of antenatal, labour and delivery, child health or sexual and reproductive health services attendance. For each indicator, the data were divided into pre-COVID (July 2019-March 2020), lockdown (April-June 2020) and post-COVID lockdown (July-December 2020). We used descriptive statistics to summarise demographic and clinical data and present summaries of outcomes before, during and after lockdown (the intervention) as medians and IQRs (online supplemental table 1). To identify suitable regression models for estimating the effects of lockdown, we first graphically plotted the number of events per month over time and assessed for stationarity and autocorrelation using the Durbin-Watson test, graphs of residuals from ordinary least square regression and graphs of auto and partial autocorrelation functions. We then conducted interrupted time series analyses using the generalised least square approach, which allows for inclusion of autoregressive or moving average autocorrelation processes. These models were used to estimate the effects of lockdown on preventative, curative, labour and delivery and child health services at KNRH. The model included a time variable (month), a dummy lockdown variable indicating prelockdown, lockdown and postlockdown and trend variables for the lockdown and postlockdown periods. This approach allows for estimation of the change in levels and trends of the outcomes following the multiple interruptions (start and lifting of lockdown). We use a $5 \%$ significance level and 95\% CIs.

\section{RESULTS}

Between 1 July 2019 and 31 December 2020, there were 14401 ANC attendances, 33499 deliveries, 111658 attendances for childhood services and 57174 sexual and reproductive health (SRH) service attendances at KNRH. There was complete closure of all antenatal, sexual health and vaccination services for the first 4 weeks of the lockdown (from 23 March 2020 to 21 April 2020). An overview of the results of the interrupted time series analysis is displayed in online supplemental table 2.

\section{Pregnancy}

\section{Preventative services}

In the 9 months prior to lockdown at the end of March 2020 , the median number of attendances for antenatal services was 894 per month (IQR 808-1035). During the 3 months of lockdown, there were 539 fewer visits a month compared with prelockdown (95\% CI 195.0 to $516.3 ; \mathrm{p}=0.001$ ). After the lifting of restrictions, the overall trend was for 370 fewer attendances compared with previous periods (95\% CI 202.7 to 536.3; $\mathrm{p}=0.001$ ).

The proportion of women receiving iron supplementation, tetanus vaccination and blood pressure monitoring remained unchanged after the initial closure of services at lockdown, despite fewer women attending ANC (figure 1A-F). However, due to stockouts of intermittent antimalarial prophylaxis and folic acid supplementation prior to lockdown, there was an increase in the proportion of women receiving medication during lockdown.

The proportion of women receiving HIV testing in ANC declined by a rate of $4 \%$ (95\% CI $1.5 \%$ to $6.5 \%$ decline; $\mathrm{p}=0.01$ ) during lockdown but increased back to baseline at the end of restrictions (figure 1G). The median number of women attending prevention of mother-tochild transmission (PMTCT) of HIV services before lockdown was 113 (IQR 56-146). Following the first month of lockdown, during which time the clinic closed, the number of attendances increased slowly with a jump of 85 more visits/month (95\% CI 31.6 to $138.4 ; \mathrm{p}=0.009$ ) in the month that lockdown was lifted (figure $1 \mathrm{H}$ ).

\section{Curative services}

The median number of women being treated for high blood pressure, pre-eclampsia and eclampsia prior to lockdown was $87 /$ month (IQR 8-180). However, during the 3 months of lockdown, there was a significantly increasing trend of 218 more women/month (95\% CI 108 to $327 ; p=0.002)$ receiving treatment. There has been a declining trend of 259 women/month (95\% CI 153 to $365 ; \mathrm{p}<0.001)$ receiving treatment in the months since lockdown was lifted. There was no change in the trend of women being treated for bacterial infections throughout the study, although this is incompletely captured in EMR.

\section{Labour and delivery}

The median number of monthly deliveries was 1869 (IQR 1791-1924) before March 2020 (pre-COVID). At the time of lockdown, there were 320 more deliveries/ month (95\% CI 199 to 441; $\mathrm{p}=0.0002)$. During lockdown, there was a trend of 109 (95\% CI 55 to 163 ; p=0.002) fewer deliveries per month, although delivery trends have increased by a median of 117 (95\% CI 54 to 180; $\mathrm{p}=0.003$ ) deliveries per month since lockdown was lifted. During lockdown, there was an increase in the rate of low-birth weight infants (1.7\% increase, 95\% CI $0.6 \%$ to $2.7 \% ; \mathrm{p}=0.011$ ), and in the immediate postlockdown month, an increase in stillbirths (1\% increase, $95 \%$ CI $-2 \%$ to $4 \% ; \mathrm{p}=0.58)$ and preterm births $(6 \%$ increase, $95 \%$ CI $-3 \%$ to $15 \%$; $\mathrm{p}=0.22)$, both not significant. There 

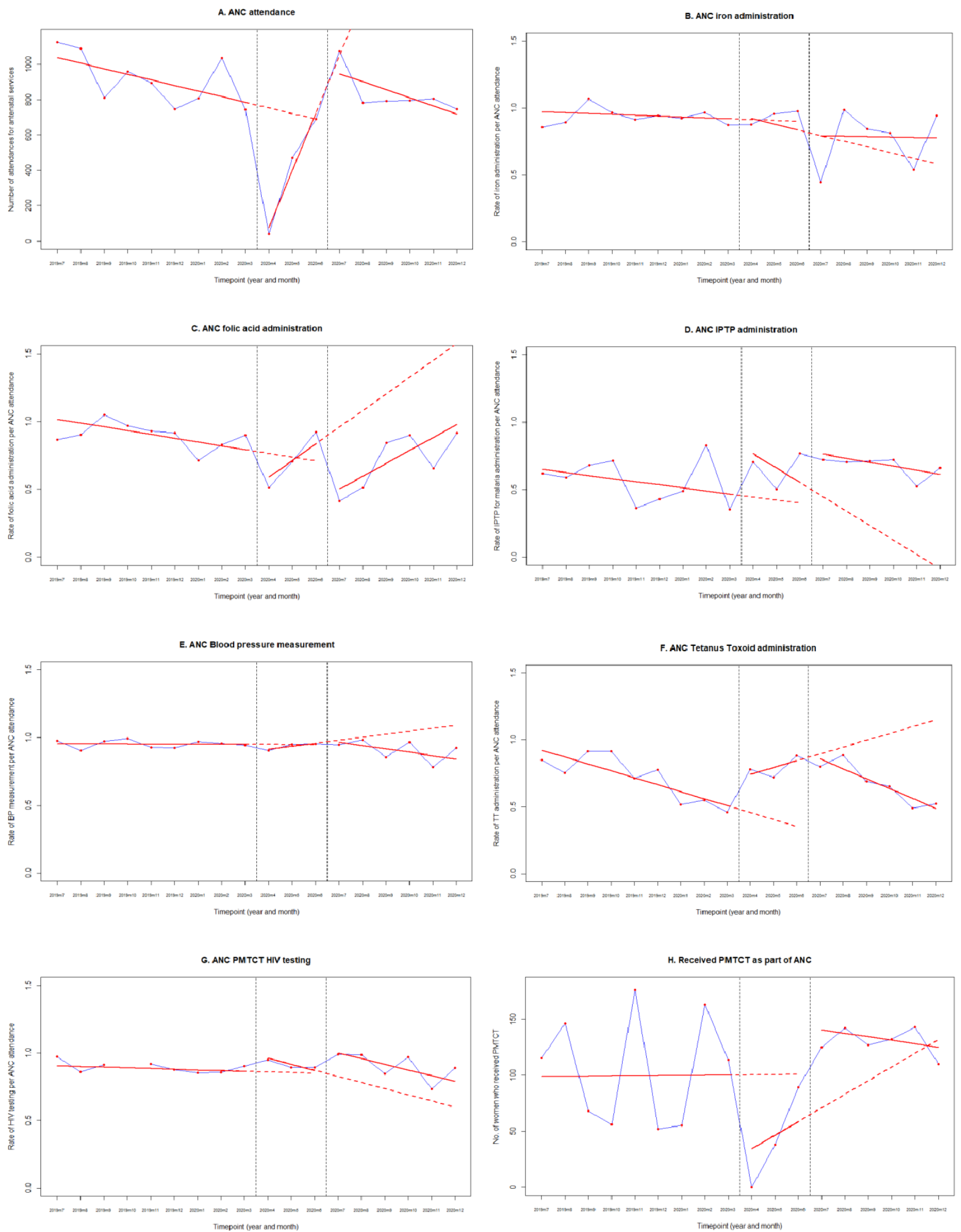

Figure 1 Antenatal indicators for Kawempe National Referral Hospital (KNRH), July 2019 to December 2020. Interrupted timeseries analyses with generalised least square regression for antenatal clinic services. (A) Antenatal clinic (ANC) attendance total attendances and rates of interventions as a proportion of total ANC attendance for: (B) ANC iron administration, (C) ANC folic acid administration, (D) ANC intermittent antimalarial prophylaxis (IPTP) administration, (E) ANC blood pressure measurement, (F) ANC tetanus toxoid administration, (G) ANC HIV testing. (H) Number of women attending prevention of mother to child transmission of HIV (PMTCT) services as part of ANC, before, during and after the COVID-19 lockdown in KNRH. Red dots represent numbers/rates per month. Blue lines connect the red dots to display the observed monthly trend. Red solid lines represent the fitted regression models. Red dashed lines represent the counterfactual scenario. 
was no increase in the rates of maternal death over the course of the study (figure 2A-G).

\section{Care of the newborn}

Prior to lockdown, there was a median of 700 admissions per month to the neonatal unit (NICU) (IQR 652-706) and a neonatal mortality rate of 39.6/1000 livebirths (IQR 34.6-50.7). During lockdown, there was an increasing rate of NICU admissions of $5.6 \%(0 \%-11 \%$; $\mathrm{p}=0.06$ ). At the end of lockdown, the neonatal mortality rate increased by 10 neonatal deaths per 1000 livebirths / month (IQR 2-10; $<<0.001$ ) (figure 3A,B).

\section{Postnatal care}

The median number of women receiving immediate routine postnatal care services (within 24 to 48 hours of delivery) pre-COVID was 1873 (IQR 1823-1993). There were no cases of COVID-19 during this study.

\section{Child health services}

Immunisations were offered on all nine scheduled immunisation days in every month in 2019 and 2020. The median number of immunisation clinic attendances in the pre-COVID period was 5871 (95\% CI 5643 to 6094). Since the lifting of lockdown, there have been 960 fewer monthly attendances (771 to 2248; $\mathrm{p}=0.04$ ) (figure $3 \mathrm{C}$ ). There was no change in the rate of children receiving Bacille Calmette-Guerin (BCG) at birth, oral polio, pneumococcal or rotavirus vaccines since the end of lockdown, although fewer children now attend the immunisation clinic. The increase in the rate of measles vaccine receipt is due to a catch-up campaign after a long stockout (online supplemental figure 1).

There was a decline in the number of children being treated for pneumonia, malaria and diarrhoea (figure 3D-F). There was an increase in the number of children treated for malnutrition after lockdown (online supplemental table 1). We do not have data on infant or child mortality during the period.

\section{Sexual and reproductive health}

There was a decrease in the number of women receiving the contraceptive pill (13.6 more women; 95\% CI 8.8 to 18.5; $\mathrm{p}<0.001)$ after restrictions were lifted. There were no changes to the number of women receiving intrauterine devices, although numbers recorded are small (figure 4A,B). The number of sterilisation procedures and number of women treated for sexually transmitted diseases remained low both pre and postlockdown. The number of abortions and sterilisations related to abortions increased by 338 (95\% CI 58 to 619 more procedures; $p=0.04$ ) at the start of lockdown.

\section{Availability of medicines}

Several shortages were noted in medication and vaccination availability both prelockdown and postlockdown, which may have affected the ability to deliver effective services (figure 5).

\section{DISCUSSION}

Despite calls for the prioritisation of antenatal services and the consideration of the indirect impacts of lockdown restrictions on maternal health, ${ }^{9} 122930$ the data from our study highlight that maternity, sexual and reproductive health, newborn and child health services were severely affected by COVID-19 restrictions.

Similar to our findings, facilities in rural Uganda saw a drop in antenatal attendances, ${ }^{31}$ as have hospitals in Kenya, Ethiopia, Zimbabwe and Rwanda in the first months of the pandemic. ${ }^{32-39}$ The aim of preventative services is to reduce maternal and newborn morbidity and mortality and any reduction in their availability can give an indication of the potential longer term impactsincluding increased rates of maternal anaemia, puerperal sepsis, stillbirth, low birth weight, preterm birth, malaria infection, pre-eclampsia/eclampsia, mother to child transmission of HIV and neonatal tetanus. ${ }^{40}$ Our study did not see an increase in maternal mortality despite fewer ANC attendances. This could be due to more women delivering in the community, as has been reported elsewhere $;^{41}$ however, our delivery rates remained constant throughout the period of study, suggesting that there may be alternative reasons for these findings, including increased maternal and neonatal morbidity, rather than mortality.

There are many proposed reasons why the ANC service and immunisation clinic attendances decreased so drastically during the lockdown. The national guidance at the start of the pandemic resulted in the closure of public transport, which a large proportion of patients rely on to access healthcare facilities, hence impacting their physical ability to access care, as has been reported in Uganda ${ }^{31} 42$ and in other countries. ${ }^{32} 363841$ Other themes which have been reported to have affected attendances are the lack of healthcare staff, fear of infection, disruption of services due to COVID-19, lockdown orders restricting movement and the increased price of transport. ${ }^{35} 43$ These themes have been highlighted in other studies in the region, ${ }^{31} 3842$ indicating the need to consider the implications of lockdown measures on public confidence in healthcare in future emergencies. The impacts of such public health measures must also be considered as to how they impact within different socioeconomic contexts, as changes to service use during the pandemic have not been universal, impacting more on those in lower socioeconomic circumstances. ${ }^{43}$

While the number of ANC visits decreased, our delivery rate did not decline by the same amount. Kawempe hospital caters for a population of 2 million people, yet the number of women attending four ANC visits remains below $90 \%$, although the majority of women in Kampala still deliver in hospital (94\%). ${ }^{44}$ This data suggest that ANC and hospital delivery are not seen as a continuum of care in our setting and could account for the phenomenon of increased deliveries despite fewer ANC visits. Alternatively, fear of contracting COVID-19 in 
BMJ Global Health

a
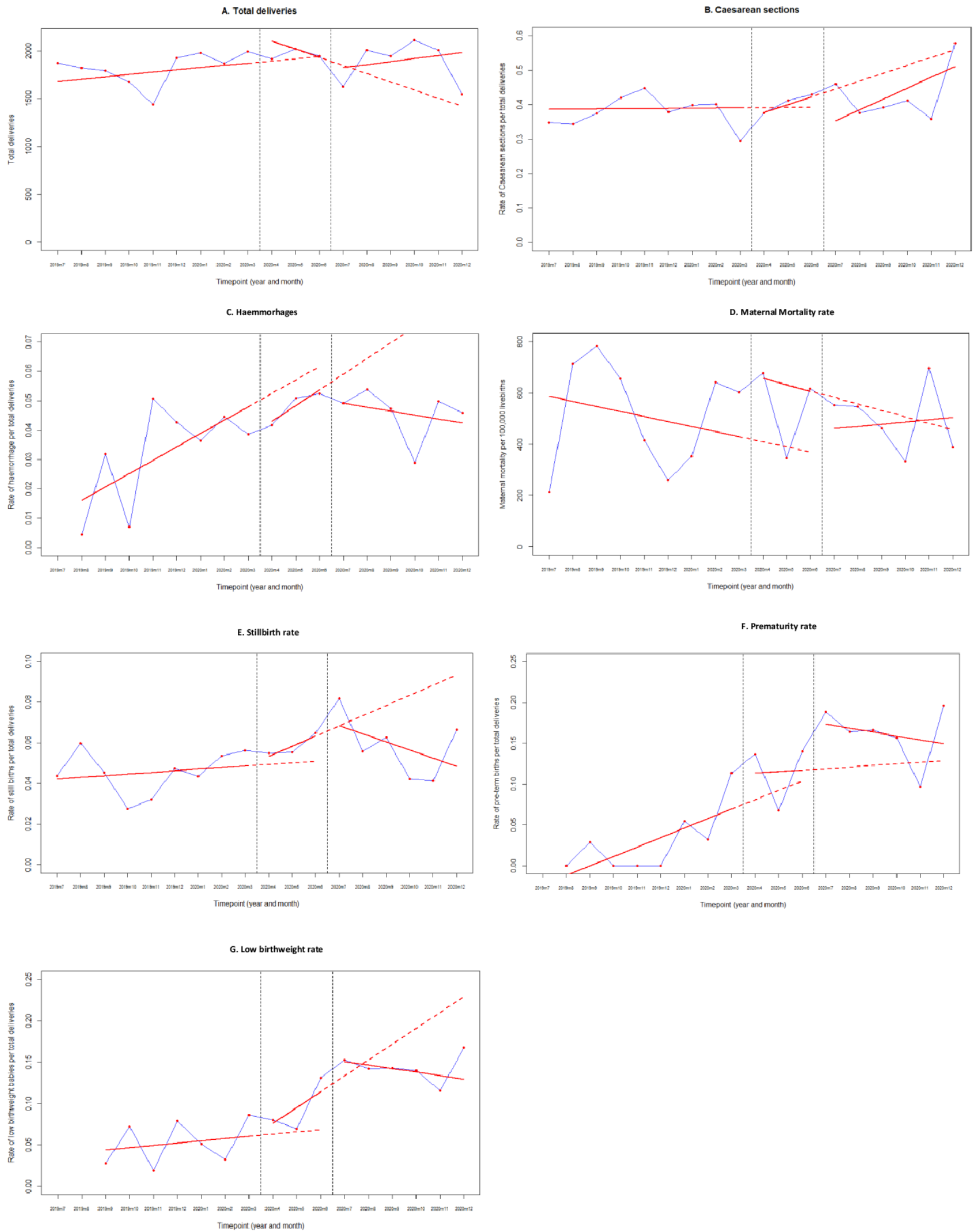

Figure 2 Labour and delivery indicators for Kawempe National Referral Hospital (KNRH), July 2019 to December 2020. Interrupted time-series analyses with generalised least square regression for (A) total deliveries, (B) caesarean sections, (C) haemorrhage related to labour and delivery, (D) maternal mortality, expressed as rate per 100000 maternities, (E) stillbirth rate as a percentage of deliveries, $(F)$ preterm birth rate as a percentage of total livebirths, $(G)$ low birth weight as a percentage of total livebirths, before, during and after the COVID-19 lockdown in KNRH. Red dots represent numbers/rates per month. Blue lines connect the red dots to display the observed monthly trend. Red solid lines represent the fitted regression models. Red dashed lines represent the counterfactual scenario.

6

Burt JF, et al. BMJ Global Health 2021;6:e006102. doi:10.1136/bmjgh-2021-006102 

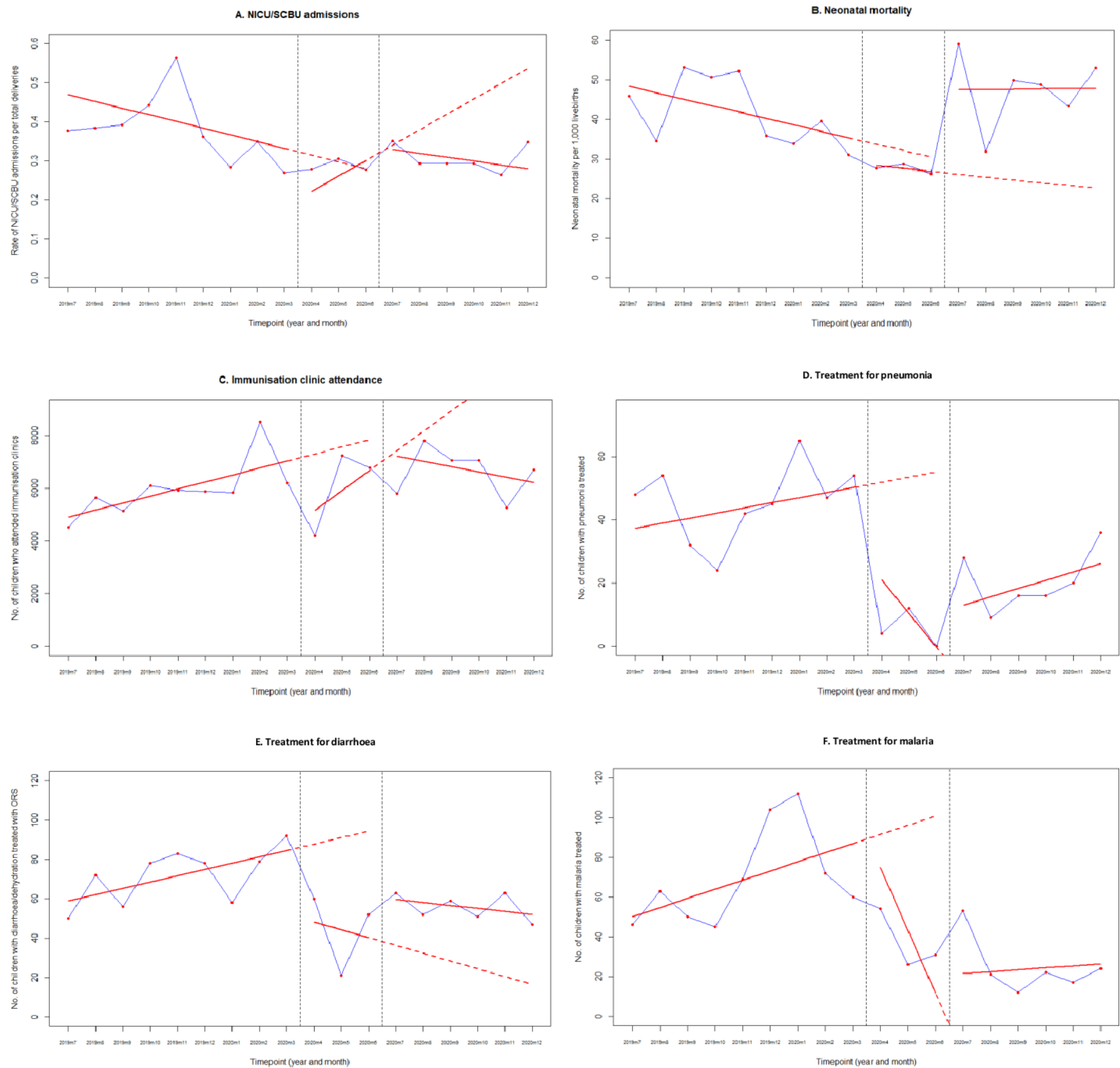

Figure 3 Neonatal and child health indicators for Kawempe National Referral Hospital (KNRH), July 2019 to December 2020. Interrupted time-series analyses with generalised least squaresregression for (A) neonatal intensive care unit admissions, (B) neonatal mortality rate expressed a rate per 1000 livebirths, (C) immunisation clinic attendance, (D) inpatient pneumonia treatment, (E) inpatient diarrhoea treatment and (F) inpatient malaria treatment, before, during and after the COVID-19 lockdown in $\mathrm{KNRH}$. Red dots represent numbers/rates per month. Blue lines connect the red dots to display the observed monthly trend. Red solid lines represent the fitted regression models. Red dashed lines represent the counterfactual scenario. NICU, neonatal intensive care unit; SCBU, special care baby unit.

the community may have influenced the decision to give birth in a hospital environment.

The COVID-19 pandemic has impacted on childbirth and deliveries across the region, ${ }^{5}{ }^{45}$ with some reports of decreased hospital deliveries. ${ }^{32-34}$ Facilities in Kenya have reported an increase in the number and rate of $\mathrm{C}$ sections and fresh stillbirths, ${ }^{36}$ increased $\mathrm{PPH}^{33}$ and an increase in maternal deaths, disproportionately affecting adolescents. ${ }^{36}$ While an increase in stillbirth and preterm birth was seen in our population at the end of lockdown, this was not significant. The age of the pregnant woman was not included in our data set and may shed a valuable light on any population-level disparities in outcomes. Similar to other studies from Africa, ${ }^{32} 34384143$ we advocate for prioritising safe and effective antenatal, intrapartum and postnatal in for future health emergencies.

The rise in neonatal deaths, low-birth-weight babies and NICU admissions are likely a result of the lack of 

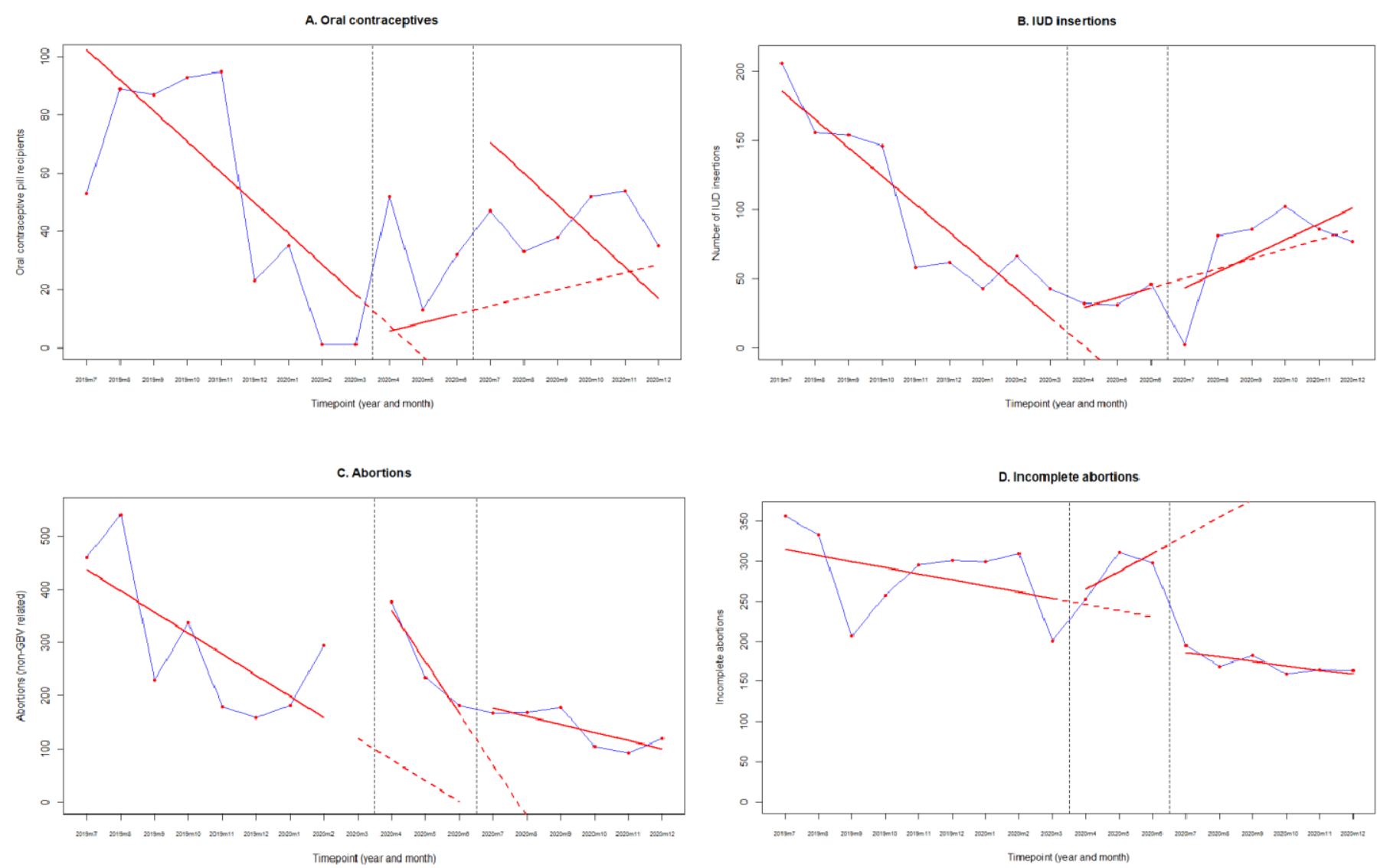

Figure 4 Sexual and reproductive health service indicators for Kawempe National Referral Hospital (KNRH), July 2019 to December 2020. Interrupted time-series analyses with generalised least square regression for (A) oral contraceptive recipients, (B) Intrauterine Devices (IUD) insertions, (C) abortions and (D) incomplete abortions, before, during and after the COVID-19 lockdown in KNRH. Red dots represent numbers/rates per month. Blue lines connect the red dots to display the observed monthly trend. Red solid lines represent the fitted regression models. Red dashed lines represent the counterfactual scenario.

ANC from March to May. ${ }^{40}$ Sudden sharp changes in neonatal outcomes have been reported in South Africa, where an increase in neonatal mortality was linked to the disruption of services and diversion of resources due to COVID-19 necessities. ${ }^{46}$ As seen with our data, a hospital in Malawi found an increase in babies born earlier and at lower birth weights, however, the same study did not find this in a Zimbabwean hospital, ${ }^{47}$ suggesting there are differences between countries that remain unexplained.

A decrease in children attending hospital, as seen in our findings, was also seen in South Africa ${ }^{46}$ and Ethiopia. ${ }^{39}$ This is likely also associated with fear of attending healthcare settings, inaccessibility, and a reduction in self-referrals, as seen with ANC..$^{39}$ Conversely, there was an increase in malnutrition attendances, likely due to the societal impacts of COVID-19 restrictions on child health and nutrition. ${ }^{48}$ The lack of therapeutic foods available may have been affected by border closures and trade restrictions, in a similar manner to medication availability in Nigeria. ${ }^{49}$

The reduction in immunisation clinic attendances in our cohort puts an estimated 20000 children at risk of mortality from vaccine-preventable diseases such as tetanus and polio. ${ }^{50}$ Uganda was declared polio-free in

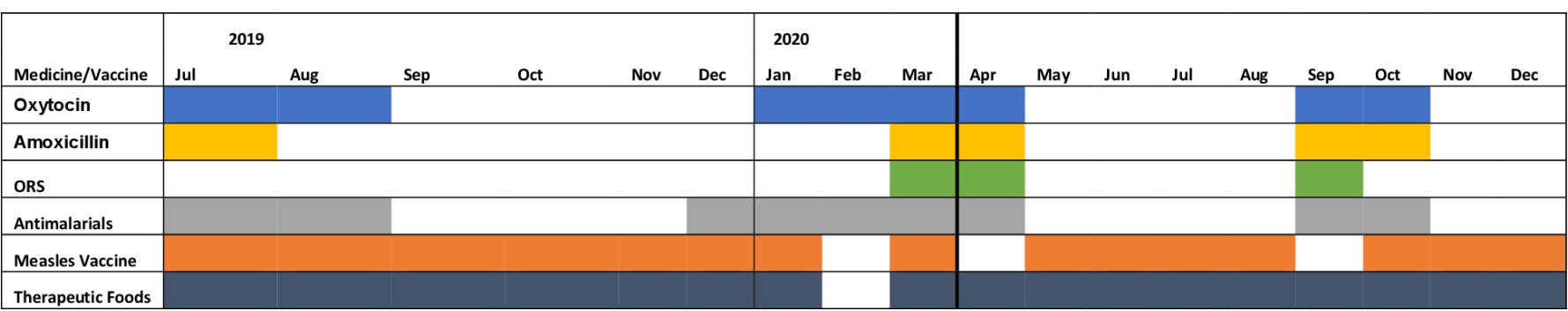

Figure 5 Medicine and vaccine unavailability in the prelockdown and postlockdown periods. Overview of medicine stockouts by month. Coloured blocks indicate no stocks available, black lines indicate start and end of lockdown. ORS, oral rehydration salts 
$2010 .^{51}$ However, the situation remains precarious due to the possibility of imported virus from surrounding countries where polio is not yet irradicated..$^{52}$ The likelihood of a potential infectious disease outbreak can be predicted based on the proportion of coverage lost by a period of reduction in immunisations, and The WHO estimates that at least 80 million children will be at risk of diseases like tetanus, polio, diphtheria and measles due to disruption of vaccination programmes during the pandemic. ${ }^{53}$ A reduction in the uptake of childhood immunisations was also seen in England, ${ }^{54}$ Singapore $^{55}$ and Brazil $^{56}$ at the start of the pandemic, highlighting the universal impact of COVID-19 on child health. The follow-up response to vaccine catch-up in this pandemic is of key importance in mitigating future outbreaks and further impacts on child health. ${ }^{5758}$

While our data show some resiliency in sexual health and contraceptive services, the reduction in HIV clinic attendances is worrying and requires further outreach work to ensure the provision of care. PMTCT services bounced back quickly, in part due to international funding support mechanisms in place, such as that from United States Agency for International Development (USAID) ${ }^{59}$ Sexual and reproductive health services have also been impacted across other East African nations. Facilities in Kenya and Ethiopia reported that contraceptive services were limited and decreases were seen in family planning attendances due to the closure of services, ${ }^{34} 39$ although national data from Kenya show no change overall in the usage of services. ${ }^{36}$

\section{Clinical and research implications}

While the effects of COVID-19 are wide ranging, the continuation of changes to antenatal services, maternal and neonatal outcomes and reduced number of children being treated for pneumonia and malaria in hospital through to December 2020 may also be influenced by other social factors. Many staff and patients were affected by restrictions to movement in October and November 2020 due to political campaigning and riots relating to the presidential elections. This highlights the susceptibility of health and healthcare services to wider events, reinforcing the need for resilience and planning going forward.

\section{Strengths and limitations}

Although the limitations of this study lie in the use of data from a single site, collected retrospectively, this has allowed the inclusion of over 33000 births, 14401 antenatal attendances and 111658 childhood immunisations, highlighting the massive impact on this population. Furthermore, the use of data from EMR rather than direct patient records mean these data are likely an under-representation of the true values of each indicator. Statistical comparison using data from the full year of 2019 would have enabled a better understanding of how 2020 compared with the time before COVID-19. Even with these documented limitations, our findings reinforce the importance of considering maternal and child health in future pandemic responses.

\section{CONCLUSIONS}

Maternal, neonatal, child and sexual and reproductive health services were all impacted by the restrictions imposed by the Ugandan government in response to COVID-19. Our results demonstrate the urgent need for pandemic responses that take into account the local context, where a stringent lockdown may be detrimental to the overall health of the population. Such responses must include the prioritisation of preventative care, including maintaining antenatal clinic visits, child health and vaccination services to prevent delayed impacts on maternal, neonatal and child health. Furthermore, any disruptions to immunisation schedules must be mitigated as rapidly as possible, to prevent further infectious disease outbreaks and future pandemics.

\section{Author affiliations}

${ }^{1}$ School of Medicine, University of Leeds, Leeds, UK

${ }^{2}$ Makerere University Johns Hopkins University, Kampala, Uganda

${ }^{3} \mathrm{MRC} / \mathrm{UVRI}$ and LSHTM Uganda Research Unit, Entebbe, Wakiso, Uganda

${ }^{4}$ Obstetrics and Gynecology, Makerere University and Mulago National Referral Hospital, Kampala, Uganda

${ }^{5}$ Infection and Immunity, St. George's, University of London, London, UK

Twitter Jessica Florence Burt @Jessica42187146

Acknowledgements We would like to thank the electronic medical records team at KNRH for access to the data for this study.

Contributors JFB, J0 and KLD developed the original research idea and drafted the manuscript, LL developed the statistics for this study, LA and AA collected and cleaned data, MS, AN, EN, PM, RM and AK all had input into the final manuscript.

Funding This publication was produced by periCOVID Africa which is part of the EDCTP2 programme supported by the European Union (grant number RIA2020EF2926 periCOVID Africa). The views and opinions of authors expressed herein do not necessarily state or reflect those of EDCTP.

Competing interests None declared.

Patient consent for publication Not required.

Ethics approval This study received ethical approval from the School of Medicine Research Ethics (SOMREC 2020-148), Committee Uganda Council for Science and Technology (HS913ES).

Provenance and peer review Not commissioned; externally peer reviewed.

Data availability statement Data are available in a public, open access repository.

Supplemental material This content has been supplied by the author(s). It has not been vetted by BMJ Publishing Group Limited (BMJ) and may not have been peer-reviewed. Any opinions or recommendations discussed are solely those of the author(s) and are not endorsed by BMJ. BMJ disclaims all liability and responsibility arising from any reliance placed on the content. Where the content includes any translated material, BMJ does not warrant the accuracy and reliability of the translations (including but not limited to local regulations, clinical guidelines, terminology, drug names and drug dosages), and is not responsible for any error and/or omissions arising from translation and adaptation or otherwise.

Open access This is an open access article distributed in accordance with the Creative Commons Attribution Non Commercial (CC BY-NC 4.0) license, which permits others to distribute, remix, adapt, build upon this work non-commercially, and license their derivative works on different terms, provided the original work is properly cited, appropriate credit is given, any changes made indicated, and the use is non-commercial. See: http://creativecommons.org/licenses/by-nc/4.0/. 


\section{REFERENCES}

1 COVID-19 response in the world Health organization African region 2020.

2 World Health Organization. The coronavirus disease 2019 (COVID-19) strategic preparedness and response plan for the WHO Africa region: 1 February 2021 - 31 January 2022. World Health Organization, 2021.

3 Uganda: Johns Hopkins University \& Medicine, 2021. Available: https://coronavirus.jhu.edu/region/uganda [Accessed 06 Jul 2021].

4 Colombo S, Scuccato R, Fadda A, et al. COVID-19 in Africa: the little we know and the lot we ignore. Epidemiol Prev 2020;44:408-22.

5 Khalil A, Kalafat E, Benlioglu C, et al. SARS-CoV-2 infection in pregnancy: a systematic review and meta-analysis of clinical features and pregnancy outcomes. EClinicalMedicine 2020;25:100446.

6 Vousden N, Bunch K, Morris E. The incidence, characteristics and outcomes of pregnant women hospitalized with symptomatic and asymptomatic SARS-CoV-2 infection in the UK from March to September 2020: a national cohort study using the UK obstetric surveillance system (UKOSS). medRxiv2021:2021.01.04.21249195.

7 Kotlar B, Gerson E, Petrillo S, et al. The impact of the COVID-19 pandemic on maternal and perinatal health: a scoping review. Reprod Health 2021;18:10.

8 Mullins E, Hudak ML, Banerjee J, et al. Pregnancy and neonatal outcomes of COVID-19: coreporting of common outcomes from PAN-COVID and AAP-SONPM registries. Ultrasound Obstet Gynecol 2021;57:573-81.

9 Roberton T, Carter ED, Chou VB, et al. Early estimates of the indirect effects of the COVID-19 pandemic on maternal and child mortality in low-income and middle-income countries: a modelling study. Lancet Glob Health 2020;8:e901-8.

10 World Health Organization. Pulse survey on continuity of essential health services during the COVID-19 pandemic. interim report. Geneva, 2020.

11 Riley T, Sully E, Ahmed Z, et al. Estimates of the potential impact of the COVID-19 pandemic on sexual and reproductive health in low- and middle-income countries. Int Perspect Sex Reprod Health 2020;46:73-6.

12 Menendez C, Gonzalez R, Donnay F, et al. Avoiding indirect effects of COVID-19 on maternal and child health. Lancet Glob Health 2020;8:e863-4.

13 Ministry of Health, ICAP, PEPFAR, US CDC, Uganda Virus Research Institute, Uganda Bureau of Statistics, WHO Uganda, UNAIDS. Uganda population-based HIV impact assessment UPHIA 20162017, 2017.

14 Busch-Hallen J, Walters D, Rowe S, et al. Impact of COVID-19 on maternal and child health. Lancet Glob Health 2020;8:e1257.

15 Vu Hoang D, Cashin J, Gribble K, et al. Misalignment of global COVID-19 breastfeeding and newborn care guidelines with World Health organization recommendations. BMJ Nutr Prev Health 2020;3:339-50.

16 Reinders S, Alva A, Huicho L, et al. Indigenous communities' responses to the COVID-19 pandemic and consequences for maternal and neonatal health in remote Peruvian Amazon: a qualitative study based on routine programme supervision. BMJ Open 2020;10:e044197.

17 Goyal M, Singh P, Singh K, et al. The effect of the COVID-19 pandemic on maternal health due to delay in seeking health care: experience from a tertiary center. Int $J$ Gynaecol Obstet 2021;152:231-5

18 Umviligihozo G, Mupfumi L, Sonela N, et al. Sub-Saharan Africa preparedness and response to the COVID-19 pandemic: a perspective of early career African scientists. Wellcome Open Res 2020;5:163.

19 Makoni M. Africa prepares for coronavirus. The Lancet 2020;395:483

20 Ministry of Health. Timeline COVID-19 response Info hub: Ministry of health, 2020. Available: https://covid19.gou.go.ug/timeline.html [Accessed 14 Jan 2021].

21 Mbabazi FK, Yahaya G, Awichi R. A mathematical model approach for prevention and intervention measures of the COVID-19 pandemic in Uganda 2020

22 Hale T, Angrist N, Cameron-Blake E. Oxford COVID-19 government response Tracker. Blavatnik School of Government, 2020.

23 Pallangyo E, Nakate MG, Maina R, et al. The impact of covid-19 on midwives' practice in Kenya, Uganda and Tanzania: a reflective account. Midwifery 2020;89:102775-75.

24 Kyohere M, Davies HG, Musoke P, et al. Seroepidemiology of maternally-derived antibody against Group B Streptococcus (GBS) in Mulago/Kawempe Hospitals Uganda - PROGRESS GBS. Gates Open Res 2020;4:155.
25 Mehring - Le Doare K. periCOVID in Africa: the periCOVID Research Group, 2021. Available: https://www.pericovid.com/pericovid-inafrica [Accessed 11 Apr 2021].

26 Aceng JR. Uganda national eHealth strategy 2017-2021. in: health RoUMo. 126. Ministry of Health.

27 Kirsty LD. Indirect effects of COVID-19 on maternal, neonatal, child, sexual and reproductive health services in Kampala, Uganda 2021.

28 Heidari S, Babor TF, De Castro P, et al. Sex and gender equity in research: rationale for the SAGER guidelines and recommended use. Research Integrity and Peer Review 2016;1:2.

29 Maternal and newborn health and COVID-19: UNICEF, 2020. Available: https://data.unicef.org/topic/maternal-health/covid-19/

30 Ogunkola IO, Adebisi YA, Imo UF, et al. Impact of COVID-19 pandemic on antenatal healthcare services in sub-Saharan Africa. Public Health Pract 2021;2:100076.

31 The Independent. Antenatal visits, deliveries drop in health facilities in Teso, 2020updated 14 May. Available from. Available: https:// www.independent.co.ug/antenatal-visits-deliveries-drop-in-healthfacilities-in-teso/ [Accessed 9 Feb 2021].

32 Wanyana D, Wong R, Hakizimana D. Rapid assessment on the utilization of maternal and child health services during COVID-19 in Rwanda. Public Health Action 2021;11:12-21.

33 Mwobobia JM. The repercussions of Covid-19 fight: standard media, 2020updated 1 June. Available from. Available: https://www. standardmedia.co.ke/health/article/2001373476/the-repercussionsof-covid-19-fight [Accessed 9 Feb 2021]

34 Thorne JG, Buitendyk M, Wawuda R, et al. The reproductive health Fall-out of a global pandemic. Sex Reprod Health Matters 2020;28:1763577.

35 Tadesse E. Antenatal care service utilization of pregnant women attending antenatal care in public hospitals during the COVID-19 pandemic period. Int J Womens Health 2020;12:1181-8.

36 Shikuku D, Nyaoke I, Gichuru S. Early indirect impact of COVID-19 pandemic on utilization and outcomes of reproductive, maternal, newborn, child and adolescent health services in Kenya. medRxiv 2020:2020.09.09.20191247.

37 Murewanhema G, Nyakanda MI, Madziyire MG. Restoring and maintaining robust maternity services in the COVID-19 era: a public health dilemma in Zimbabwe. Pan Afr Med J 2020;37:32.

38 Ahmed SAKS, Ajisola M, Azeem K, et al. Impact of the societal response to COVID-19 on access to healthcare for non-COVID-19 health issues in slum communities of Bangladesh, Kenya, Nigeria and Pakistan: results of pre-COVID and COVID-19 lockdown stakeholder engagements. BMJ Glob Health 2020;5:e003042.

39 Abdela SG, Berhanu AB, Ferede LM, et al. Essential healthcare services in the face of COVID-19 prevention: experiences from a referral hospital in Ethiopia. Am J Trop Med Hyg 2020;103:1198-200.

40 World Health Organization. Who recommendations on antenatal care for a positive pregnancy experience. World Health Organization, 2016.

41 Ombere SO. Access to maternal health services during the COVID-19 pandemic: experiences of indigent mothers and health care providers in Kilifi County, Kenya. Front Sociol 2021;6:613042.

42 Ogunleye OO, Basu D, Mueller D, et al. Response to the novel corona virus (COVID-19) pandemic across Africa: successes, challenges, and implications for the future. Front Pharmaco 2020;11:1205-05.

43 Temesgen K, Wakgari N, Debelo BT, et al. Maternal health care services utilization amidstCOVID-19 pandemic in West Shoa zone, central Ethiopia. PLoS One 2021;16:e0249214.

44 Uganda Bureau of Statistics, ICF. Uganda demographic and health Survery 2016. Kampala, Uganda and Rockville, Maryland, USA: Uganda Bureau of Statistics ICF, 2018: 153.

45 Chmielewska B, Barratt I, Townsend R, et al. Effects of the COVID-19 pandemic on maternal and perinatal outcomes: a systematic review and meta-analysis. Lancet Glob Health 2021;9:e759-72.

46 Jensen C, McKerrow NH. Child health services during a COVID-19 outbreak in KwaZulu-Natal Province. South Africa, 2020.

47 Chimhuya S, Neal SR, Chimhini G. Indirect impacts of the COVID-19 pandemic at two tertiary neonatal units in Zimbabwe and Malawi: an interrupted time series analysis. medRxiv2021:2021.01.06.21249322.

48 Akseer N, Kandru G, Keats EC, et al. COVID-19 pandemic and mitigation strategies: implications for maternal and child health and nutrition. Am J Clin Nutr 2020;112:251-6.

49 Akande-Sholabi W, Adebisi YA. The impact of COVID-19 pandemic on medicine security in Africa: Nigeria as a case study. Pan Afr Med $J$ 2020;35:73

50 Abbas K, Procter SR, van Zandvoort K, et al. Routine childhood immunisation during the COVID-19 pandemic in Africa: a benefit- 
risk analysis of health benefits versus excess risk of SARS-CoV-2 infection. Lancet Glob Health 2020;8:e1264-72.

51 Horn of Africa is polio-free: global polio eradication initiative, 2010 Available: https://polioeradication.org/news-post/horn-of-africa-ispolio-free/ [Accessed 11 Apr 2021]

52 AFP. South Sudan confirms outbreak of vaccine-derived polio: medical Xpress, 2020. Available: https://medicalxpress.com/ news/2020-10-south-sudan-outbreak-vaccine-derived-polio.html [Accessed 12 Apr 2021].

53 Abad-Vergara D, Sidhu S, Fulker J. At least 80 million children under one at risk of diseases such as diphtheria, measles and polio as COVID-19 disrupts routine vaccination efforts, warn Gavi. WHO and UNICEF, 2020. https://www.who.int/news/item/22-05-2020at-least-80-million-children-under-one-at-risk-of-diseases-suchas-diphtheria-measles-and-polio-as-covid-19-disrupts-routinevaccination-efforts-warn-gavi-who-and-unicef

54 McDonald HI, Tessier E, White JM, et al. Early impact of the coronavirus disease (COVID-19) pandemic and physical distancing measures on routine childhood vaccinations in England, January to April 2020. Euro Surveill 2020;25.

55 Zhong Y, Clapham HE, Aishworiya R, et al. Childhood vaccinations: hidden impact of COVID-19 on children in Singapore. Vaccine 2021;39:780-5.

56 Silveira MF, Tonial CT, Goretti K Maranhão A, et al. Missed childhood immunizations during the COVID-19 pandemic in Brazil: analyses of routine statistics and of a national household survey. Vaccine 2021;39:3404-9.

57 Masresha BG, Luce R, Shibeshi ME, et al. The performance of routine immunization in selected African countries during the first six months of the COVID-19 pandemic. Pan Afr Med J 2020;37:12.

58 Mburu CN, Ojal J, Chebet R, et al. The importance of supplementary immunisation activities to prevent measles outbreaks during the COVID-19 pandemic in Kenya. BMC Med 2021;19:35.

59 Jiang H, Zhou Y, Tang W. Maintaining HIV care during the COVID-19 pandemic. Lancet HIV 2020;7:e308-9. 
Supplementary Table 1: Results of interrupted time series analyses with generalised least squares regression for several indicators

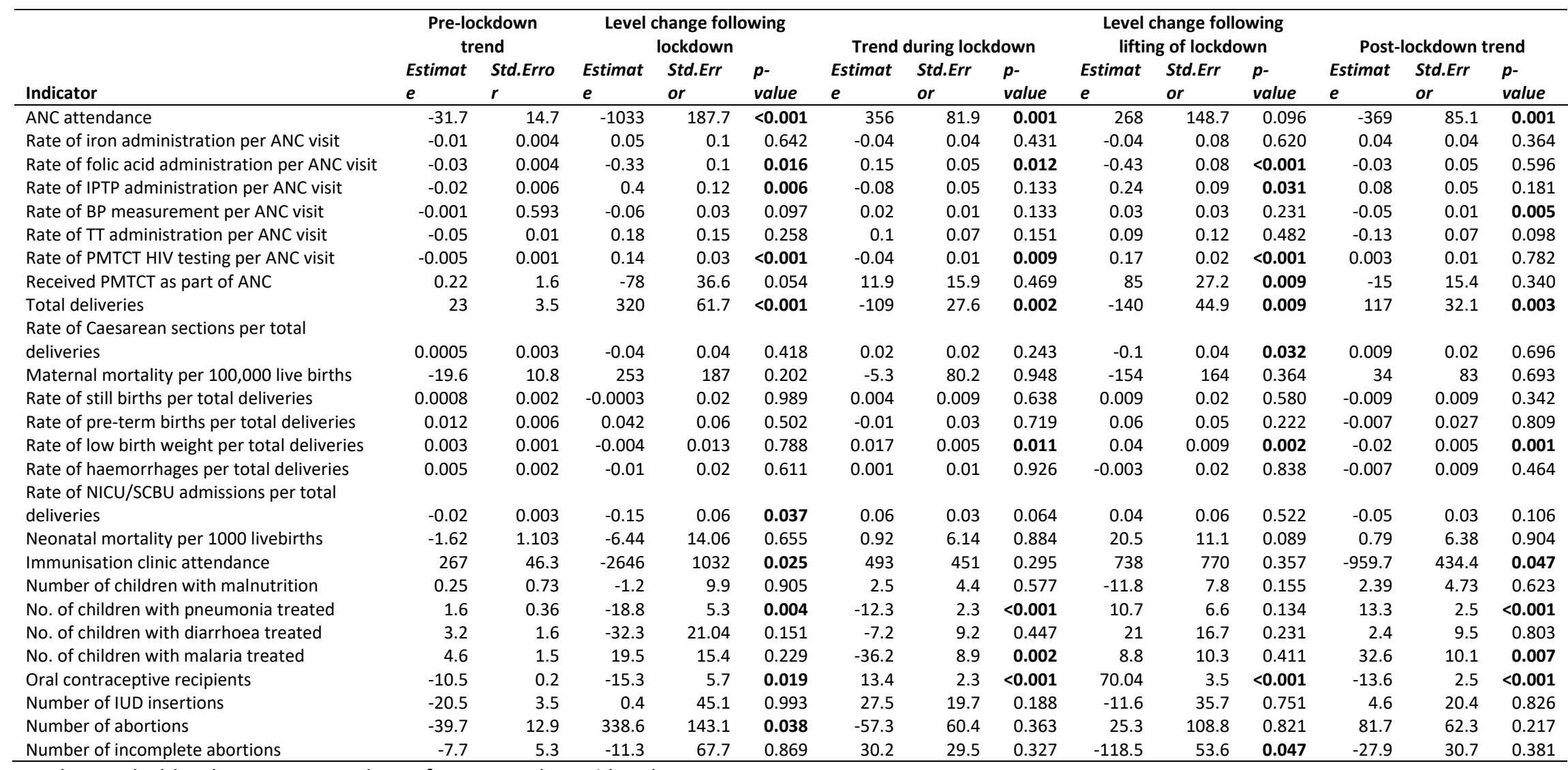

$p$-values in bold indicate statistical significance at the $5 \%$ level 
Supplementary Table 2 - median and interquartile range for measures at KNRH

\begin{tabular}{|l|l|l|l|}
\hline Measure & Pre-lockdown (IQR) & During lockdown (IQR) & Post lockdown (IQR) \\
\hline ANTENATAL SERVICES & & & \\
\hline $\begin{array}{l}\text { 1. Antenatal attendances } \\
\text { Preventative services }\end{array}$ & $894(808-1035)$ & $469(255-579)$ & $794(785-802)$ \\
\hline 2. Iron supplementation & $863(747-961)$ & $449(242-561)$ & $658(523-694)$ \\
\hline 3. Folic acid supplementation & $850(682-931)$ & $331(176-484)$ & $598(469-678)$ \\
\hline 4. Tetanus Toxoid vaccination & $683(567-819)$ & $337(185-473)$ & $533(426-656)$ \\
\hline 5. Intermittent antimalarial prophylaxis & $551(325-687)$ & $235(132-382)$ & $560(507-573)$ \\
\hline 6. Blood pressure measurement & $823(783-984)$ & $446(242-552)$ & $729(681-769)$ \\
\hline 7. HIV testing and counselling & $781(686-899)$ & $419(229-518)$ & $722(665-773)$ \\
\hline 8. Prevention of mother to child transmission services & $113(56-146)$ & $38(19-64)$ & $130(126-140)$ \\
\hline Curative services & & & \\
\hline 9. Hypertension, pre-eclampsia, eclampsia & $87(8-180)$ & $357(179-365)$ & $255(147-344)$ \\
\hline 10. Bacterial infection & $39(25-53)$ & $47(45-48)$ & $41(31-50)$ \\
\hline LABOUR AND DELIVERY & & & $1977(1706-2009)$ \\
\hline 11. Total deliveries & $1869(1791-1924)$ & $1944(1931-1984)$ & $10.2(38.0-44.8)$ \\
\hline 12. Caesarean section rate (\% deliveries) & $37.8(34.8-40.2)$ & $41.2(39.4-42.9)$ & $86(73-96)$ \\
\hline $\begin{array}{l}\text { 13. Haemorrhage associated with delivery (combined } \\
\text { ante- and postpartum) }\end{array}$ & $72(12-77)$ & $102(91-103)$ & \\
\hline 14. Maternal Death rate/100,000 women & $602(354-657)$ & $617(482-648)$ & $505(406-552)$ \\
\hline 15. Stillbirth rate (\% total deliveries) & $4.5(4.3-5.5)$ & $5.5(5.5-6.0)$ & $5.9(4.6-6.6)$ \\
\hline 16. Low birthweight infants (\% total deliveries) & $3.2(2.7-7.2)$ & $8.0(7.5-10.5)$ & $14.2(14.0-15.0)$ \\
\hline 17. Premature infant rate (\% total deliveries)* & $0(0-3.2)$ & $13.7(10.2-13.8)$ & $16.5(15.8-18.3)$ \\
\hline CARE OF THE NEWBORN & & & \\
\hline 18. Neonatal intensive care admissions & $700(652-706)$ & $540(538-579)$ & $571(546-585)$ \\
\hline 19. Neonatal mortality rate/1000 livebirths & $39.6(34.6-50.7)$ & $27.6(26.9-28.2)$ & $49.3(44.7-52.2)$ \\
\hline POSTNATAL CARE & & & \\
\hline
\end{tabular}




\begin{tabular}{|l|l|l|l|}
\hline 20. Women receiving postnatal care & $1873(1823-1993)$ & $1965(1941-2052)$ & $1929(1870-1985)$ \\
\hline CHILD HEALTH SERVICES & & & \\
\hline 21. Immunisation clinic visits & $5871(5643-6094)$ & $6781(5488-7015)$ & $6877(6017-7060)$ \\
\hline 22. Treatment for pneumonia & $47(42-54)$ & $4(2-8)$ & $18(16-26)$ \\
\hline 23. Treatment for diarrhoea & $78(58-79)$ & $52(37-56)$ & $56(51-62)$ \\
\hline 24. Treatment for malaria & $63(50-72)$ & $31(29-43)$ & $22(18-24)$ \\
\hline 25. Treatment for malnutrition** & $0(0-5)$ & $13(9-14)$ & $10(9-24)$ \\
\hline SEXUAL AND REPRODUCTIVE HEALTH SERVICES & & & \\
\hline 26. Oral contraceptive recipients & $53(23-89)$ & $32(23-42)$ & $43(36-51)$ \\
\hline 27. Injectable contraceptive recipients & $84(0-171)$ & $86(85-112)$ & $212(201-224)$ \\
\hline 28. Intrauterine devices & $66(58-154)$ & $32(32-39)$ & $84(78-86)$ \\
\hline 29. Abortions & $262(182-365)$ & $08-305)$ & $144(109-169)$ \\
\hline
\end{tabular}

*Neonatal unit closed for refurbishment prior to lockdown

** Iong term stockout of nutritional foods during much of the year prior to lockdown 

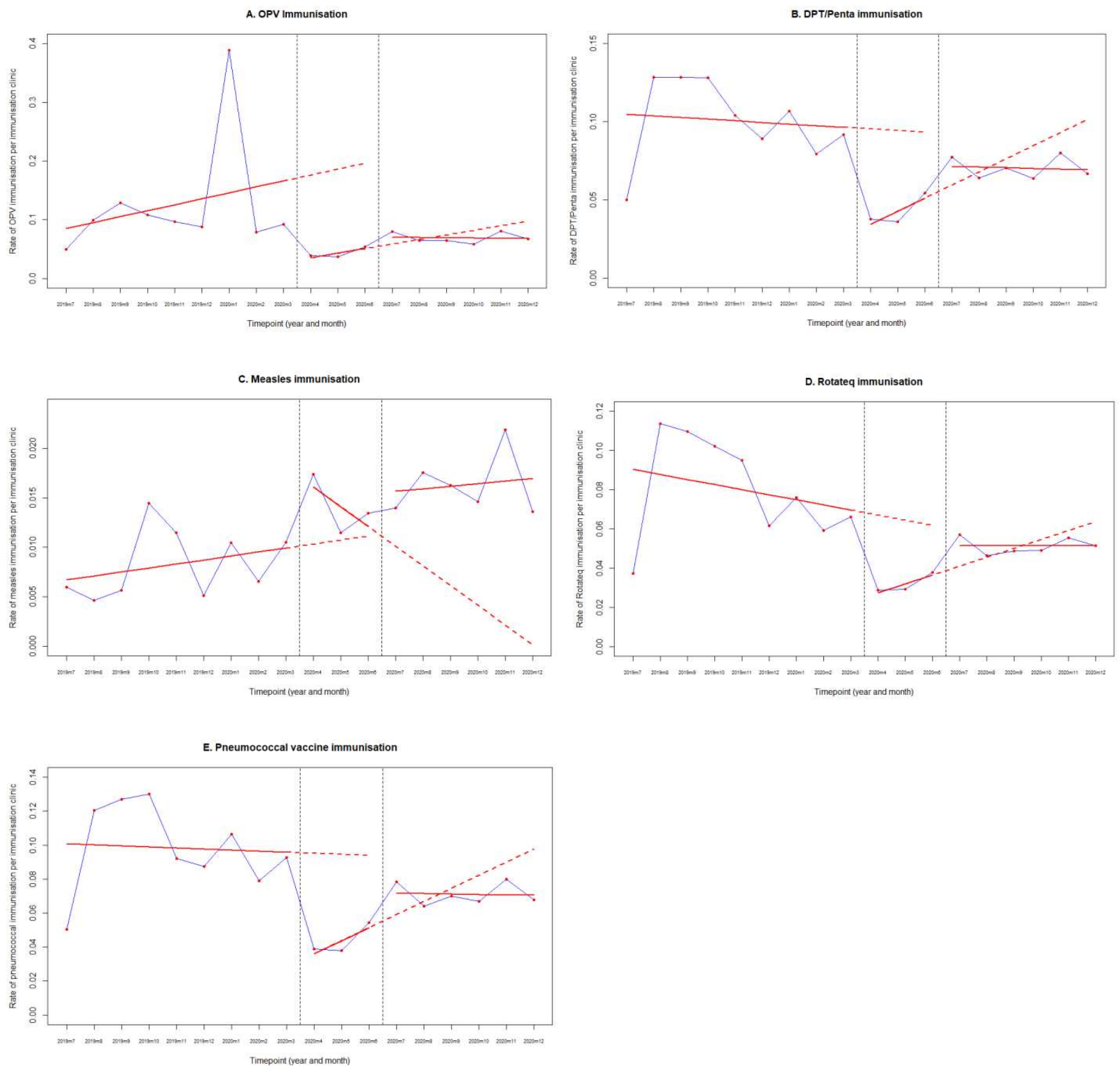